\title{
Hereditary Hemorrhagic Telangiectasia with SMAD4 Mutations Is Associated with Fatty Degeneration of the Left Ventricle, Coronary Artery Aneurysm, and Abdominal Aortic Aneurysm
}

\author{
Yasunori Inoguchi, Bunji Kaku, Naotaka Kitagawa and Shoji Katsuda
}

\begin{abstract}
:
A 52-year-old man with recurrent epistaxis and palpebral conjunctival telangiectasia visited our hospital for a follow-up checkup for gastrointestinal polyposis. At 48 years of age, he underwent Y-graft replacement for an abdominal aortic aneurysm. Arteriovenous malformation was detected in his lungs, and a genetic test revealed an SMAD4 mutation. Eventually, he was diagnosed with juvenile polyposis-hereditary hemorrhagic telangiectasia (JP-HHT) syndrome. In addition, fatty degeneration of the left ventricle and a coronary aneurysm were detected. This is the first report suggesting the possibility of an association between these manifestations and JP-HHT due to SMAD4 mutations. Examining cardiovascular disorders in JP-HHT patients is imperative.
\end{abstract}

Key words: hereditary hemorrhagic telangiectasia, JP-HHT, SMAD4, coronary aneurysm, fatty degeneration, abdominal aortic aneurysm

(Intern Med 58: 387-393, 2019)

(DOI: 10.2169/internalmedicine.1287-18)

\section{Introduction}

Hereditary hemorrhagic telangiectasia (HHT) is an autosomal-dominant inherited vascular malformation syndrome characterized by telangiectases and arteriovenous malformation (AVM) that reportedly occurs in 1 in 10,000 individuals (1). HHT is characterized by recurrent and severe nosebleeds, mucocutaneous telangiectases, and visceral (pulmonary, cerebral, hepatic, and spinal) AVM. The occurrence of HHT is attributed to genetic mutations that modulate transforming growth factor-beta (TGF- $\beta$ ) signaling. Reportedly, mutations have been detected in endoglin (ENG), activin A receptor type II-like 1 (ACVRL1/ALK1), SMAD4, and bone morphogenetic protein 9 (2). Previously, an SMAD4 mutation was detected in $<2 \%$ of cases clinically suspected of HHT, and this mutation has been reported to be related to juvenile polyposis (3). HHT is typically characterized by visceral AVM; however, whether or not malformations of the aorta and coronary are determined in HHT re- mains unclear.

We herein report the case of a patient with JP-HHT who had various cardiovascular disorders.

\section{Case Report}

A 52-year-old man had had recurrent epistaxis since he was a child. He did not suffer from Kawasaki disease. While his father had been diagnosed with a thoracic aorta aneurysm and cerebral hemorrhaging, his mother died of gastric cancer at 65 years of age. In addition, three of four children of his mother's cousins had recurrent epistaxis. At our hospital, he received medical treatment for diabetes mellitus and gastrointestinal polyposis. As he had substantial gastric polyps that bled easily (Fig. 1), he was taking an iron drug to improve his iron-deficiency anemia. Although he had multiple polyps throughout his colon, the polyps were not malignant, as confirmed by a biopsy.

At 48 years of age, he underwent Y-graft replacement for an abdominal aorta aneurysm (Fig. 2). After two years, com- 
A

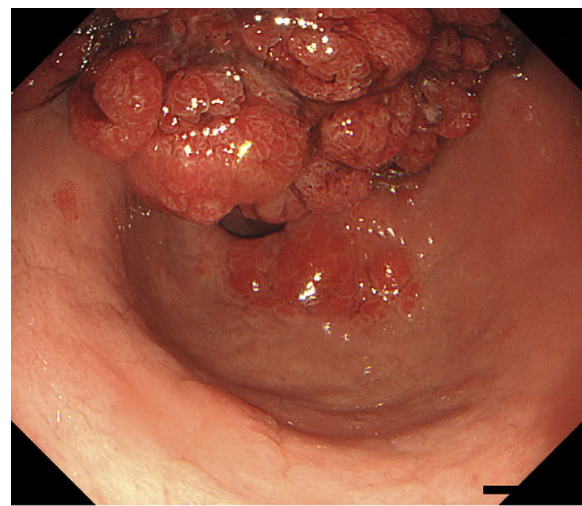

C

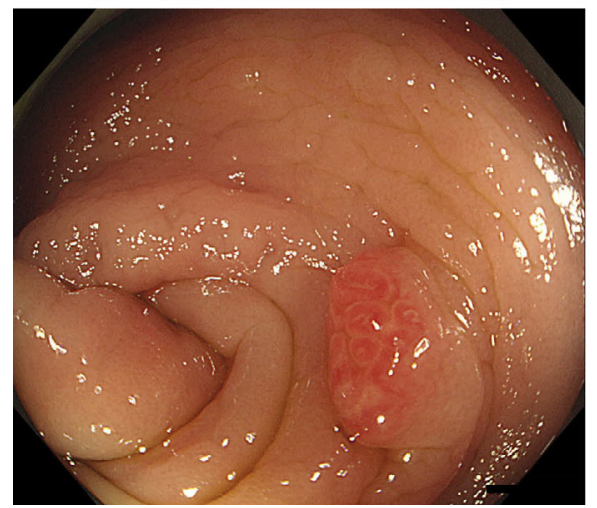

B

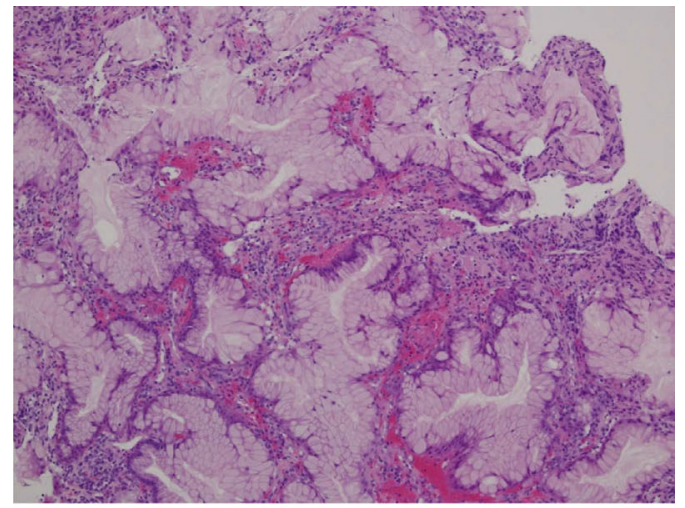

D

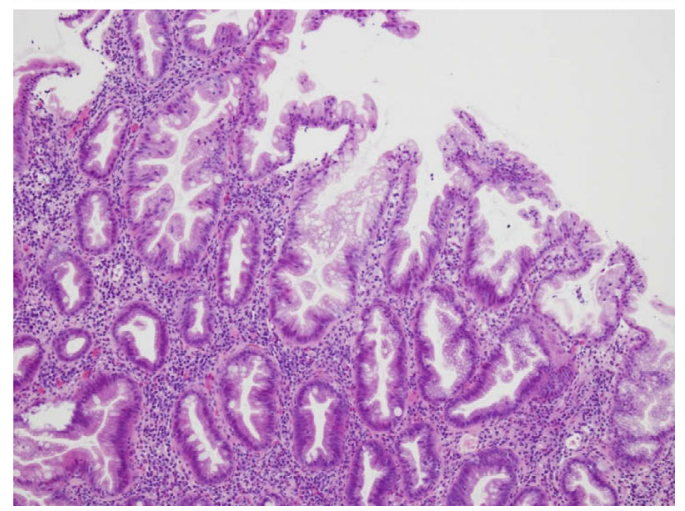

Figure 1. Gastrointestinal endoscopy and colonoscopy. A: The gastric polyps were massive and bled easily. B: Hematoxylin and Eosin (H\&E) staining of the gastric polyps. Foveolar hyperplasia and small round cells were observed (lymphocyte>plasma cell). The gastric foveola showed an increased complexity of structure and density. C: Multiple polyps were located throughout the colon. D: H\&E staining of the colon polyps. Hyperplastic polyps of the microvesicular type were observed, along with mild dysplasia of the gland duct.

A

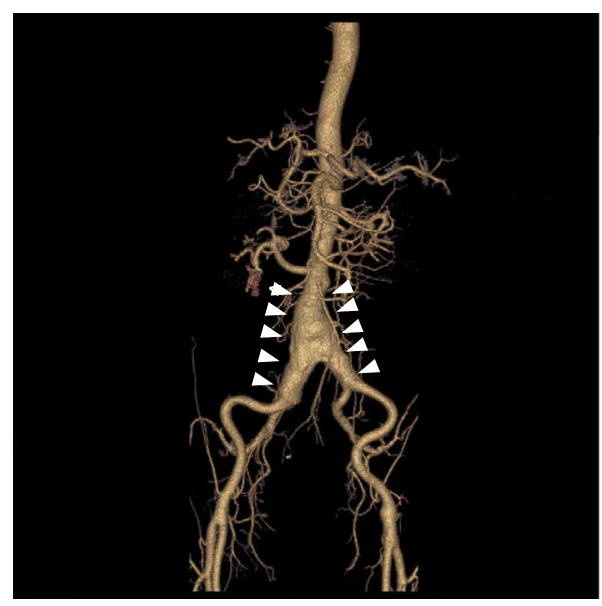

B

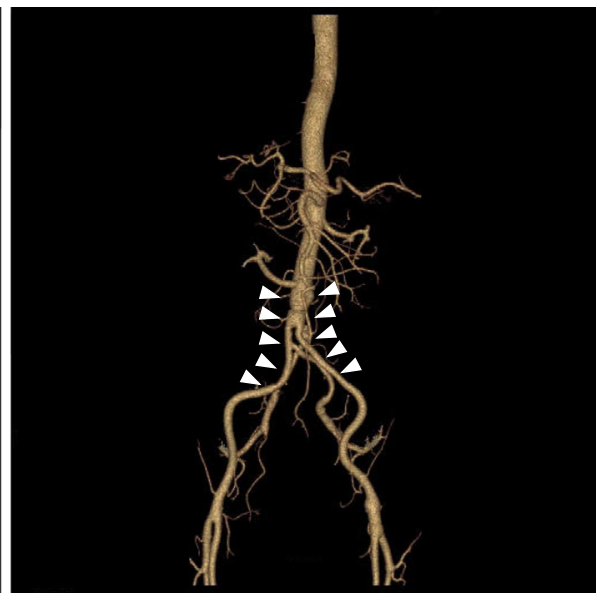

Figure 2. Three-dimensional computed tomography of the aorta. A: An aneurysm of the abdominal aorta before surgery (white arrowheads). B: Y-graft replacement for the aneurysm of the abdominal aorta (white arrowheads).

puted tomography performed to follow the aneurysm postoperatively revealed two AVMs in the lingular segment of the left lung (Fig. 3). In addition, he exhibited telangiectasia of the palpebral conjunctiva and finger clubbing. Based on his clinical history, including symptoms of epistaxis, palpebral conjunctival telangiectasia, and AVMs detected in the lung, HHT was suspected, so we conducted a genetic test. As the genetic test revealed an SMAD4 mutation with a frameshift in exon 10 (Fig. 4), he was diagnosed with HHT accompanied by juvenile polyposis (JP-HHT).

As he later complained of exertional dyspnea and his symptoms gradually worsened, he was referred to the divi- 

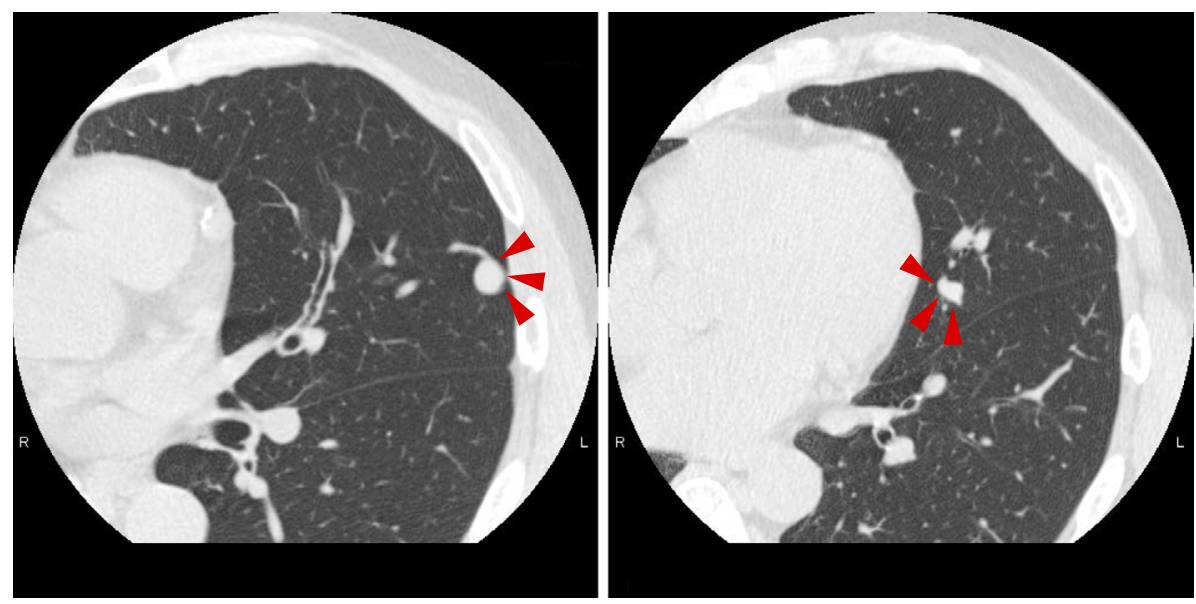

Figure 3. Computed tomography of the lungs. Two AVMs are present in the lingular segment of the left lung (red arrowheads). Coil embolization was subsequently performed by interventional radiology.

\begin{tabular}{|l|l|l|l|l|}
\hline Gene & Region & $\begin{array}{l}\text { Change of } \\
\text { DNA sequence }\end{array}$ & $\begin{array}{l}\text { Change of } \\
\text { amino acid sequence }\end{array}$ & Significance \\
\hline SMAD4 & exon10 & c.1245-1248delCAGA & p.Asp415Glufs*20 & $\begin{array}{l}\text { Pathologic } \\
\text { variation }\end{array}$ \\
\hline
\end{tabular}

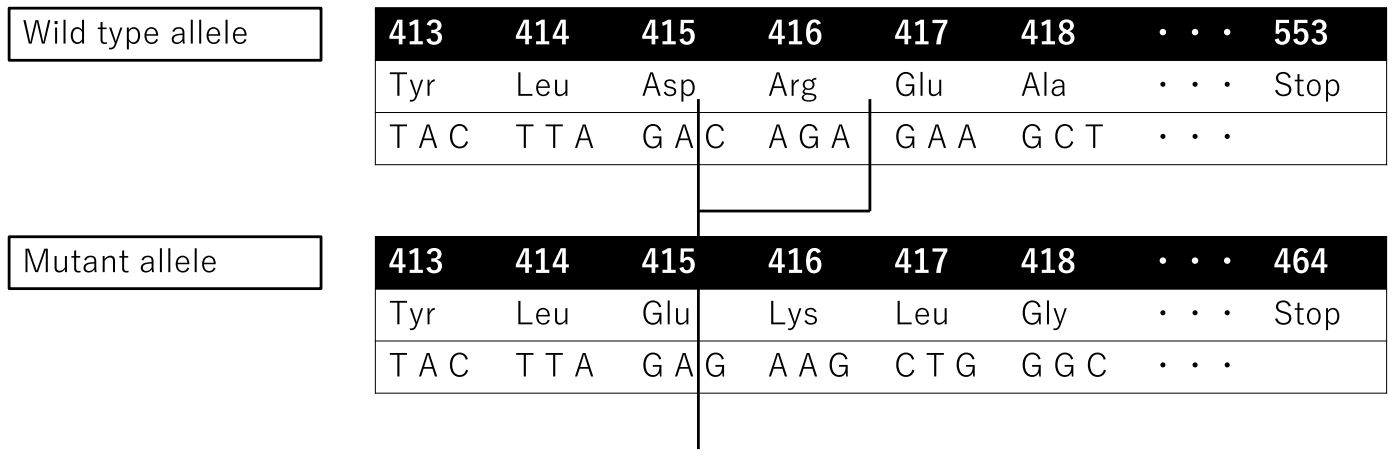

Figure 4. The genetic analysis results. The SMAD4 mutation in our case was a frameshift of exon 10. As the mutated gene had four deleted bases, the reading frame of the codon was shifted, inducing premature termination of translation.

sion of cardiovascular medicine to assess his cardiovascular system. Laboratory test results revealed marginal microcytic anemia and low serum levels of ferritin and iron; however, these findings resulted in no marked changes in his clinical course. His serum brain natriuretic peptide value was normal, and his angiotensin-converting enzyme level was slightly low. In addition, his electrocardiography results were normal, and chest X-ray revealed slight cardiomegaly but no congestion in the lungs or pleural effusion. Echocardiography suggested that the inferior and posterior walls of the left ventricle (LV) were hyperechoic and hypokinetic; however, myocardial thinning or hypertrophy was not detected in other regions. The LV ejection fraction was $49 \%$. Although mild mitral regurgitation (MR) was detected, the aortic, pulmonary, and tricuspid valves revealed no apparent findings. Technetium tetrofosmin myocardial perfusion scintigraphy revealed a perfusion abnormality in the inferior, posterior, and anteroseptal walls of the LV. Cardiac magnetic resonance imaging revealed fatty degeneration of the inferior and posterior walls of the LV, revealing a low signal in the fat-suppressed T2-weighted image and late gadolinium enhancement of the anteroseptal wall (Fig. 5). Furthermore, coronary angiography revealed an aneurysm of the left and right coronary arteries, which were highly tortuous and dilated (Fig. 6).

The patient's oxygen concentration in the arterial blood was $88.8 \mathrm{mmHg}$ at rest, which decreased to $52.5 \mathrm{mmHg}$ after exertion. We therefore held the AVMs in the lung accountable for exertional dyspnea and performed coil embolization of the AVMs accordingly. Regarding the treatment plan, we did not use antiplatelet medicine because of the easy bleeding from the gastric and colon polyps. We administered losartan because of an aortic disorder and the gene mutation in the TGF- $\beta$ signal pathway. 

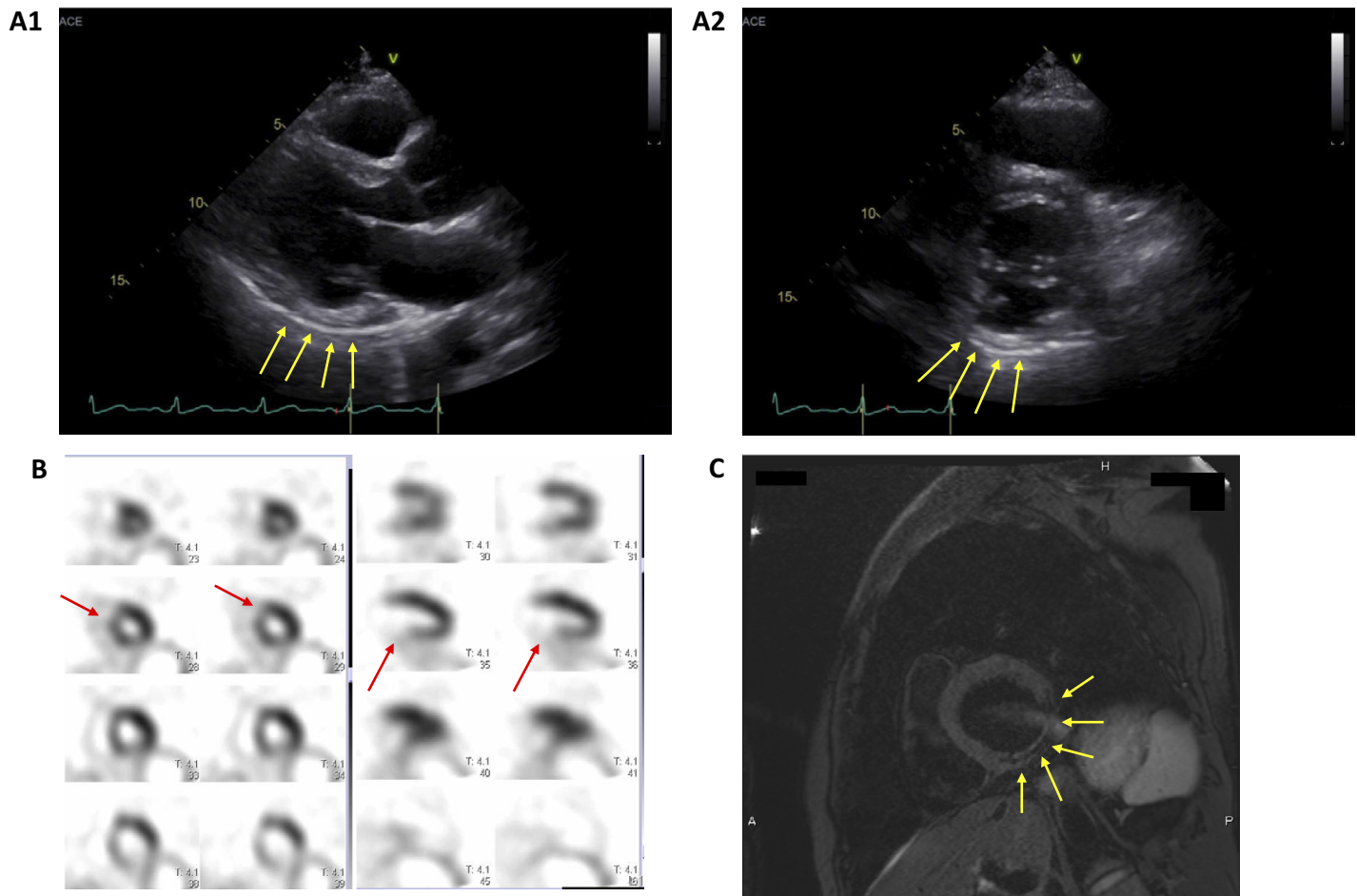

Figure 5. Imaging findings of the left ventricle (LV). A1 and A2: Echocardiography revealed that the inferior and posterior walls of the $\mathrm{LV}$ were hyperechoic and hypokinetic (yellow arrows). The $\mathrm{LV}$ ejection fraction was $49 \%$. While mild mitral regurgitation was found, the aortic, pulmonary, and tricuspid valves showed no remarkable findings. B: Myocardial perfusion scintigraphy revealed a perfusion abnormality in the inferior, posterior, and anteroseptal walls of the LV (red arrows). C: Cardiac MRI revealed fatty degeneration of the inferior and posterior walls of the $L \mathrm{~V}$, showing a low signal on fat-suppressed T2-weighted imaging (yellow arrows).

\section{Discussion}

HHT is an autosomal-dominant inherited vascular malformation syndrome characterized by telangiectases and AVMs. The leading clinical manifestation of HHT is spontaneous and recurrent epistaxis because of any of the following: telangiectases of the nasal mucosa; dermal, oral, and gastrointestinal telangiectases; solid-organ AVMs, especially of the lungs, liver, and brain; or a family history of the same. HHT should therefore be considered in individuals with any of these characteristics, including epistaxis, mucocutaneous telangiectases, AVMs, and a family history. While the presence of two of the above features suggests HHT, three or more of these features confirm the diagnosis of HHT (4). Of note, the leading presenting symptom is epistaxis, which occurs in $>90 \%$ of patients and first manifests at an average of 12 years of age. Typically, the symptoms of telangiectases appear after 30 years of age and are exacerbated with age. Recurrent hemorrhaging from the gastrointestinal tract is reportedly a standard feature in the later years in $15-20 \%$ of individuals (5).

Genetic tests can diagnose HHT, as several genes associated with it have been detected even in cases where the symptoms for the diagnosis are inadequate. Two decades ago, an ENG gene mutation was reported (6) followed by an
ACVRL1/ALK1 mutation (7). An SMAD4 gene mutation has also been detected (3). These gene mutations occur in the TGF- $\beta$ signaling pathway, which regulates cell proliferation, differentiation, apoptosis, and migration; it is apparent that these mutations are associated with cardiovascular disease (8).

In the present case, we diagnosed the patient with JPHHT, although JP and HHT are known to be clinically diverse diseases. While JP is caused by mutations in SMAD4 and bone morphogenetic protein receptor type-1A, HHT is caused by mutations in ENG and ACVRL1/ALK1 (9). In addition, ENG and ACVRL1/ALK1 mutations are detected in approximately $85 \%$ of cases clinically suspected of being HHT. Recent studies have reported that SMAD4 mutations cause both JP and HHT, rendering JP-HHT a well-known disease $(3,9)$. As an SMAD4 mutation is detected in $<2 \%$ of cases clinically suspected of being HHT (3), the detection of an SMAD4 mutation in patients with HHT is rare. Nevertheless, screening patients with HHT who have an SMAD4 mutation for JP is imperative, due to the increased risk of JP-associated gastrointestinal cancer in such patients (10). In contrast, JP patients with SMAD4 mutations should be considered at risk of developing visceral manifestations of HHT with high morbidity $(3,9,10)$. Furthermore, in our case, the patient's initial symptom was only gastrointestinal polyposis; however, a characteristic symptom of HHT became apparent 


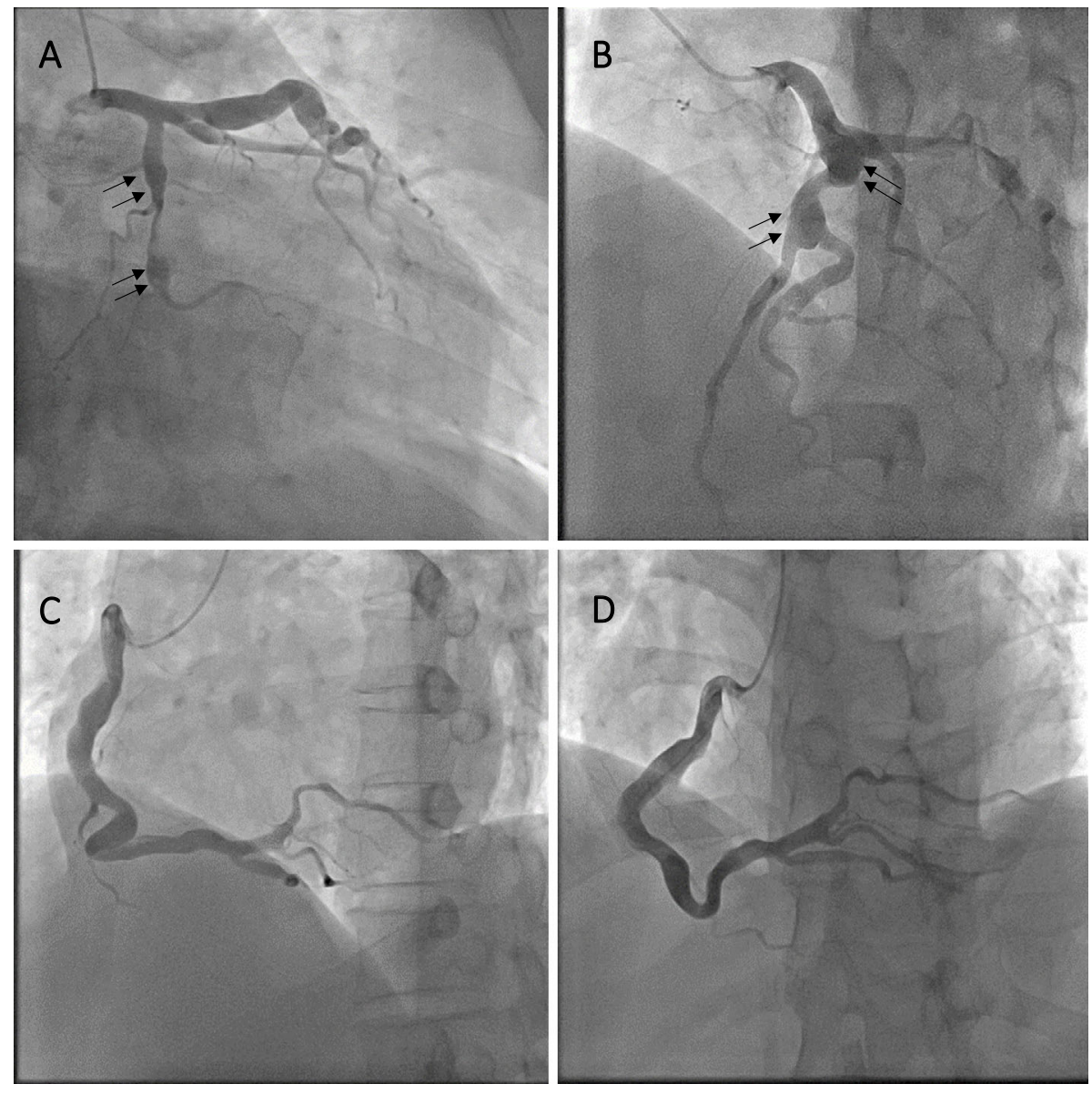

Figure 6. Coronary angiography. A: The RAO/caudal view revealed an aneurysm of the left circumflex artery (black arrows). B: The LAO/cranial view revealed an aneurysm of the left descending artery (black arrows). $\mathrm{C}$ and D: The right coronary artery was highly tortuous and dilated.

during the course. Ultimately, a genetic test diagnosed HHT caused by an SMAD4 mutation.

In our case, the patient suffered from an abdominal aortic aneurysm at a relatively young age (48 years). After that, he underwent Y-graft replacement for an abdominal aorta aneurysm. Although the occurrence of an aortic disorder in patients with HHT harboring SMAD4 mutations is extremely rare, such cases have been reported several times in the literature. Teekakirikul et al. (11) reported two cases of JPHHT (case 1: 11-year-old boy; case 2: 34-year-old woman). Both cases had aortic dilation, and the father of case 2 died suddenly at 60 years of age from acute aortic dissection and rupture of the ascending aorta. In addition, Heald et al. (12) conducted a retrospective chart review to ascertain the prevalence of aortic disorders in an HHT cohort. They identified patients from a single institution who met the Curacao Criteria for the clinical diagnosis of HHT and assessed 26 patients from 15 families (1 ACVRL1, 4 ENG, 8 SMAD4, and 2 clinical diagnoses). Their findings revealed that 6 of $26(23 \%)$ patients had an aortic disorder, and all had SMAD 4 mutations. As 6 of 16 (38\%) SMAD4 mutation carriers had an aortic disorder in their cohort, the rate of aortic disorders in SMAD4 mutation carriers was high. Thus, the authors believed that these data suggested an aortic disorder could be part of the characterization of SMAD4-induced HHT.

SMAD4 interacts in the same signal transduction pathway as TGF- $\beta$ receptors 1 and 2 (TGF- $\beta$ R $1 / 2$ ) and fibrillin- 1 (FBN1), mutations of which cause aortic disorders (13). SMAD4 mutations may therefore cause aortic disorders, such as Marfan syndrome (MFS) and Loeys-Dietz syndrome (LDS). Wain et al. (14) reported that $21 \%$ of their patients (7/34) with SMAD4 mutations exhibited features suggestive of a connective tissue defect. Indeed, several studies have reported cases of aortic disorder with an SMAD4 gene mutations $(11,12)$. In contrast, only a few studies have reported ENG and ACVRL1 mutations in cases of aortic disorders. In addition, patients with ENG or ACVRL1 gene mutations present more frequently with pulmonary hypertension than aortic disorder, particularly in carriers with ACVRL1 (15).

Although our case did not demonstrate mitral valve prolapse (MVP) or severe MR, it did reveal that enhanced TGF- $\beta$ signaling contributed to myxomatous changes in the mitral and tricuspid valves (13). Furthermore, MVP and severe MR are more common in patients with MFS than carriers with other mutations in the TGF- $\beta$ signaling pathway. All genes associated with HHT are part of the TGF- $\beta$ signaling pathway, which is vital for the vascular integrity and 
angiogenic remodeling (14). In particular, as several studies have suggested a correlation between SMAD4 mutations and aortic disorders, the presence of SMAD4 mutations should be assessed in cases of cardiovascular disease that involves the coronary artery.

However, few studies have explored the correlation between HHT and coronary artery disease. Mieghem et al. (16) reported a 33-year-old woman with HHT who suffered from spontaneous coronary artery dissection. His et al. (17) reported a 50-year-old man with HHT who had a large ascending aortic aneurysm and giant coronary artery aneurysms involving the right, left main, left anterior descending, and circumflex coronary arteries. However, neither report described any gene mutations of HHT, making ours the first report that suggests the possibility that a patient with HHT harboring an SMAD4 mutation could have a coronary aneurysm.

As patients with HHT are in a state of hypercoagulability because of elevated plasma levels of factor-VIII, they might also suffer from thrombotic complications (18). We should therefore consider administering anticoagulant therapy when we detect a coronary artery aneurysm in patients with HHT. In our case, as the factor-VIII plasma levels were normal and the patient's chronic anemia could be attributed to gastric polyps that easily bled, we did not perform anticoagulant therapy.

To our knowledge, fatty degeneration of the LV has not been reported. TGF- $\beta$ appears to be responsible for cardiac fibrosis, cardiomyocyte apoptosis, and cardiac hypertrophy (19), making it a crucial regulator of cardiac remodeling through its direct and potent actions in mediating the cardiomyocyte growth, fibroblast activation, and extracellular matrix deposition (8). This is the first report to suggest the possibility of fatty degeneration of the LV.

Furthermore, the renin-angiotensin system (RAS) plays a vital role in cardiac remodeling, and clinical trials have documented the efficacy of angiotensin II inhibition in patients with myocardial infarction and heart failure. Angiotensin can induce TGF- $\beta$ activation through its induction of thrombospondin. As TGF- $\beta$ has been suggested to act downstream of angiotensin signaling, RAS inhibitors are considered effective against diseases involving disorders of the TGF- $\beta$ signaling pathway. Indeed, losartan is beneficial for MFS (20). Losartan reportedly decreases the aortic root dilatation rate by blocking angiotensin II receptor type I and reducing the TGF- $\beta$ production (20). Based on these previous findings and because our present case had SMAD4 mutations in the TGF- $\beta$ signaling pathway, we administered losartan. In Japan, Sumitomo et al. (21) reported the first case of JP-HHT caused by an SMAD4 mutation in 2013. However, this report is the first concerning cardiovascular disorders in a patient with JP-HHT caused by SMAD4 mutations in Japan.

This report has some limitations. First, only the patient was examined by a genetic test because his other family members declined to undergo the test. In addition, his fam- ily members also did not undergo cardiac imaging tests, such as coronary angiography, cardiac magnetic resonance imaging, or myocardial perfusion scintigraphy. We therefore cannot prove that SMAD4 mutations cosegregate with coronary aneurysms and fatty degeneration of the LV. A coronary aneurysm is typically caused by trauma, Kawasaki disease, iatrogenic, infection, connective tissue disease, or vasculitis; however, the clinical course, physical findings, laboratory data, and computed tomography in our patient ruled out these causes. Second, mutations in the ACVRL1, ENG, and GDF2 genes were not detected; we did not examine genes other than those relevant to HHT. In other words, it is impossible to deny that this patient had other gene mutations that might have caused his condition. We therefore cannot exclude the possibility that some unknown factors or mutations other than the SMAD4 gene might have caused the coronary aneurysm and fatty degeneration of the inferior and posterior walls of the LV. However, as already mentioned, SMAD4 is involved in the TGF- $\beta$ signaling pathway, the mutations of which not only cause various cardiovascular disorders but are also involved in the myocardial structure itself, such as cardiac remodeling. There is therefore a high probability that the SMAD4 mutations in the present case contributed to his coronary aneurysm and fatty degeneration of the LV.

We hope that the observations in this case report will prove helpful in the treatment and examination of patients with JP-HHT harboring SMAD4 mutations.

\section{Conclusion}

We herein report a JP-HHT case with SMAD4 mutations with various cardiovascular complications accompanied by multiple symptoms of cardiovascular disorders, such as a coronary artery aneurysm, fatty degeneration of the LV, and an abdominal aorta aneurysm. This report shows that SMAD 4 mutations can cause aortic disorders, as SMAD4 is involved in the TGF- $\beta$ signaling pathway, which causes several diseases. Given that symptoms of HHT with SMAD4 mutations can extend to coronary artery abnormalities, evaluating the systemic vasculature should always be considered.

\section{The authors state that they have no Conflict of Interest (COI).}

\section{References}

1. Marchuk DA, Guttmacher AE, Penner JA, et al. Report on the workshop on hereditary hemorrhagic telangiectasia, July 10-11, 1997. Am J Med Genet 76: 269-273, 1998.

2. McDonald J, Wooderchak-Donahue W, Webb CV, et al. Hereditary hemorrhagic telangiectasia: genetics and molecular diagnostics in a new era. Front Genet 6: 1, 2015.

3. Gallione CJ, Repetto GM, Legius E, et al. A combined syndrome of juvenile polyposis and hereditary haemorrhagic telangiectasia associated with mutations in MADH4 (SMAD4). Lancet 363: 852859, 2004.

4. Shovlin CL, Guttmacher AE, Buscarini E, et al. Diagnostic criteria 
for hereditary hemorrhagic telangiectasia (Rendu-Osler-Weber syndrome). Am J of Med Genet 91: 66-67, 2000.

5. Govani FS, Shovlin CL. Hereditary haemorrhagic telangiectasia: a clinical and scientific review. Eur J Hum Genet 17: 860-871, 2009.

6. McAllister KA, Grogg KM, Johnson DW, et al. Endoglin, a TGFbeta binding protein of endothelial cells, is the gene for hereditary haemorrhagic telangiectasia type 1. Nat Genet 8: 345-351, 1994.

7. Johnson DW, Berg JN, Baldwin MA, et al. Mutations in the activin receptor-like kinase 1 gene in hereditary haemorrhagic telangiectasia type 2. Nat Genet 13: 189-195, 1996.

8. Goumans MJ, Liu Z, Dijke PT, et al. TGF- $\beta$ signaling in vascular biology and dysfunction. Cell Res 19: 116-127, 2009.

9. Gallione C, Aylsworth AS, Beis J, et al. Overlapping spectra of SMAD4 mutations in juvenile polyposis (JP) and JP-HHT syndrome. Am J Med Genet A 152A: 333-339, 2010

10. Gallione CJ, Richards JA, Letteboer TGW, et al. SMAD4 mutations found in unselected HHT patients. J Med Genet 43: 793-797, 2006.

11. Teekakirikul P, Milewicz DM, Miller DT, et al. Thoracic aortic disease in two patients with juvenile polyposis syndrome and SMAD4 mutations. Am J Med Genet A 161A: 185-191, 2013.

12. Heald B, Rigelsky C, Moran R, et al. Prevalence of thoracic aortopathy in patients with juvenile polyposis syndrome-hereditary hemorrhagic telangiectasia due to SMAD4. Am J Med Genet A 167A: 1758-1762, 2015.

13. Andrabi S, Bekheirnia MR, Patricia Robbins-Furman, et al. SMAD4 mutation segregating in a family with juvenile polyposis, aortopathy, and mitral valve dysfunction. Am J of Med Genet A 155A: 1165-1169, 2011.
14. Wain KE, Ellingson MS, McDonald J, et al. Appreciating the broad clinical features of SMAD4 mutation carriers: a multicenter chart view. Genet Med 16: 588-593, 2014.

15. Vorselaars VMM, Velthuis S, Snijder RJ, et al. Pulmonary hypertension in hereditary haemorrhagic telangiectasia. World J Cardiol 7: 230-237, 2015.

16. Mieghem CAGV, Ligthart JMR, Cademartiri F. Spontaneous dissection of the left main coronary artery in a patient with Osler Weber Rendu disease. Heart 92: 394, 2006.

17. His DH, Ryan GF, Hellems SO, et al. Large aneurysms of the ascending aorta and major coronary arteries in a patient with hereditary hemorrhagic telangiectasia. Mayo Clin Proc 78: 774-776, 2003.

18. Dittus C, Streiff M, Ansell J. Bleeding and clotting in hereditary hemorrhagic telangiectasia. World J Clin Cases 3: 330-337, 2015.

19. Liu G, Ma C, Yang H, et al. Transforming growth factor $\beta$ and its role in heart disease. Exp Ther Med 13: 2123-2128, 2017.

20. Pepe G, Giusti B, Sticchi E, et al. Marfan syndrome: current perspectives. Appl Clin Genet 9: 55-65, 2016.

21. Sumitomo Y, Yamashita Y, Itai R, et al. A case of combined syndrome of juvenile polyposis and hereditary hemorrhagic telangiectasia associated with SMAD4 mutation. Nihon Shokakibyo Gakkai Zasshi (Jpn J Gastroenterol) 110: 64-73, 2013 (in Japanese, Abstract in English).

The Internal Medicine is an Open Access journal distributed under the Creative Commons Attribution-NonCommercial-NoDerivatives 4.0 International License. To view the details of this license, please visit (https://creativecommons.org/licenses/ by-nc-nd/4.0/).

(C) 2019 The Japanese Society of Internal Medicine Intern Med 58: 387-393, 2019 